\title{
Comparative evaluation of antidiabetic activity of Rosmarinus officinalis $L$. and Chamomile recutita in streptozotocin induced diabetic rats
}

\author{
Manal A Emam \\ Biochemistry Department, Faculty of Science, Ain Shams University, Cairo, Egypt \\ Corresponding author: kheffha@yahoo.com. \\ ABSTRACT

\begin{abstract}
Herbal formulations are getting more importance in the treatment of diabetes, cancer and hepatic disorder because of the hazardous adverse effects of the current therapy. Especially diabetes can be controlled by Allopathic medicine as well as Herbal medicine. A comparison was made between the antidiabetic activities of water extracts of leaves of Rosmarinus Officinalist and Chamomile recutitain Streptozotocin- induced diabetic rats. Serum Fasting blood glucose and other biochemical parameters such as total protein; carbohydrate hydrolyzing enzyme; $\alpha$ amylase, total antioxidant capacity, liversteoteosis; total cholesterol, Triacylglycerol, were determined at the dose $200 \mathrm{mg} / \mathrm{kg}$ body weight for 21 days. There is a significant increase decrease in serum fasting blood glucose $(p<0.001)$, increase in body weight and improvement in the other biochemical parameters with treatment of herbal formulations which altered in diabetic rats There was a significant decrease in serum glucose level $(p<0.001)$, increase in body weight and changes in normal cells was observed with treatment of the above mentioned extracts which altered in diabetic rats as compared to the control rats standard.
\end{abstract}

Key words: Rosmarinus Officinalist, Chamomile recutita, antidiabetic activity

\section{INTRODUCTION}

Diabetes mellitus is a metabolic disorder characterized by hyperglycemic, glucoseurea and negative nitrogen balance and it is mainly due to lack of insulin secretion in beta cells of pancreas and desensitization of insulin receptors for insulin. It is the most prevalent disease in the world affecting $25 \%$ of population and afflicts 150 million people and is set to rise to 300 million by 2025 (Vats et al., 2005). It causes number of complications like retinopathy, neuropathy, and peripheral vascular insufficiencies (Chehadeet al., 2005). Diabetes is still not completely curable by the present anti diabetic agents. Insulin therapy is the only satisfactory approach in diabetic mellitus, even though it has several drawbacks like insulin resistance (Piedrolaet al., 2001), anorexia, brain atrophy and fatty liver in chronic treatment (Weidmannet al., 1993). Herbal drugs are gaining popularity in the treatment of diabetic mellitus (Pariet al., 1999). The major advantages of herbal medicine seem to be their efficacy, low incidence of side effects, and low cost.

Rosemary, Rosmarinus officinalis L. (Labiatae) is an evergreen perennial shrub grown in many parts of the world. It has been reported to possess a number of therapeutic applications in folk medicines in curing or managing wide range of diseases such as diabetes mellitus, respiratory disorders, stomach problems and inflammatory diseases. Rosemary has long been recognized as having antioxidant molecules, such as rosmarinic acid, carnasol and rosmaridiphenol (Bakirel et al., 2008).

Chamomile tea is prepared with dried flowers from Matricaria chamomile L., and this plant has been used as an herbal medicine component in Europe. Especially, chamomile has been used to treat various inflammations, irritations, and pains such as skin diseases, wounds, eczema, ulcers, gout, neuralgia, and rheumatic pains (Srivastava and Gupta, 2007).

Furthermore, a recent study demonstrated that chamomile plant extract suppresses the growth of human cancer cells and causes apoptosis (Srivastava and Gupta, 2007). Major secondary components from $M$. chamomile belong to three different chemical classes: sesquiterpenes, coumarins, and flavonoides (McKay and Blumberg, 2006). The major components of the essential oil are (-)-R-bisabolol and R-farnesene, and the yield of the essential oil from the flowers are about $0.4 \%$. This plant also has high levels of polyphenolic compounds such ascoumarins and flavonoides. The coumarins, herniarin, umbelliferone, and esculetin make up approximately $0.1 \%$ of the total constituents. The major flavonoides components are apigenin, luteolin, 
and quercetin, which comprise 16.8, 1.9, and 9.9\%, respectively, of total flavonoides. Thus, chamomile is one of the richest sources of dietary antioxidants. These coumarins and flavonoides are soluble in hot water, and the amounts obtained from frequent consumption of tea are not negligible. There is substantial evidence that these compounds have suppressive activity on oxidative damage to skins, membranes, proteins, and DNA by inhibiting free radical scavenging activity and contribute to protection against chronic health disorders such as atherosclerosis and hypertension.

Here we report hypoglycemic activity of the water extract from leaves of rosemary and chamomile with a view to provide scientific evidence of modern lines in Streptozotocin induced diabetic rat (SIDR) to evaluate and compare their antidiabetic activity. The effects produced by these formulations on blood glucose and other biochemical parameters were evaluated (Kato et al., 2008).

\section{MATERIAL AND METHODS}

Collection and Extraction of Plant Material: The fresh leaves of the plant Rosmarinus officinalis (Rosemary) and Matricaria chamomile $L$ (Chamomile) were obtained from the local herbal market of Kingdom Saudi Arabia. About $10 \mathrm{gm}$ of each plant leaves of rosemary and chamomile was taken in each separate glass jar and soaked in 100 of boiled water. The container with its content was occasional shaking and stirring for 1 hour, and leaved overnight at room temperature. The extracts were then filtered through filter paper (Double Rings filter paper $102,11.0 \mathrm{~cm}$ ). The filtrates were concentrated at $50^{\circ} \mathrm{C}$ under reduce pressure using vacuum pump rotary evaporator to afford a greenish mass of leaves extracts.

Experimental animals: Experiment was conducted on adult male albino rats with the weights of 150 $200 \mathrm{gm}$ procured from Animal House of Medical Research Centre, Faculty of Medicine, Ain Shams University. All rats were fed normal laboratory chow food containing $16 \%$ protein, $66 \%$ carbohydrate, $8 \%$ fats and water. All rats were housed at a $(12: 12) \mathrm{hr}$ light and dark cycle at $25^{\circ} \mathrm{C}$ and relative humidity $(60-70) \%$. The guidelines followed for animal experiment were approved by Ain Shams University committee.

Antidiabetic activity evaluation: Rats were made diabetic with the single dose of Streptozotocin (STZ)

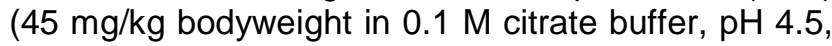
i.p.). After $72 \mathrm{hrs}$, the animals with fasting blood glucose level >200 mg/dl were considered diabetic.
The experiment was carried on 6 groups of six rats in each group to study the effect of plant water extract on STZ induced diabetes and changes in biochemical parameters as follows;

Group 1: Normal control, receive normal rat fed and water, ad libitum

Group 2: Diabetic control, STZ induced rats.

Group 3: Normal rats administered rosemary extract ( $200 \mathrm{mg} / \mathrm{kg}$ body weight).

Group 4: Normal rats administered chamomile extract (200 mg/ kg body weight).

Group 5: Diabetic test, treated with water extract of rosemary for 3 weeks after STZ injection $(200 \mathrm{mg} / \mathrm{kg}$, p.o.)

Group 6 :Diabetic test, treated with the water extract of chamomile (200 mg/kg, p.o.)

Study protocol: The treatment with plant extract (1 $\mathrm{ml}$ ) was given daily for 3 weeks using gastric cannula (Makino et al., 2002). No detectable irritation or restlessness was observed after extract administration. No noticeable adverse effect (i.e., respiratory distress, abnormal locomotion or catalepsy) was observed in any animals after the extract administration. Throughout the experimental period, the body weight, was monitored. Starting from the 1st day (3rd day of STZ-injection) of extract administration to diabetic rats, FBG level was measured in every 7th day using glucometer (Mallicket al., 2007). On the 21th day of extract administration, blood samples were collected from overnight fasted rats by cardiac puncture under mild ether anesthesia. Blood samples were kept for 30 min. and centrifuged at $3000 \mathrm{rpm}$, for $20 \mathrm{~min}$. The serum thus obtained was separated and used immediately for the further estimation.

\section{METHODS}

Streptozotocin from Sigma-Aldrich Corp. Colorimetric kits used for the estimation of total protein (TP), aamylase, total antioxidant capacity (TAC), Total Cholesterol (TC) and Triacylglycerol (TG) were obtained from Bio-diagnostic Company. All other chemicals were of high analytical grade and were obtained from Sigma-Aldrich Corp.

Statistical analysis: The data obtained in present investigation was subjected to statistical analysis. All results are expressed as Mean \pm SD. The data was analyzed using Analysis of variance (ANOVA) and the group means were compared by Dunnet test. 
Values were considered statistically significant when $\mathrm{p}<0.05$.

\section{RESULTS}

Effect on blood glucose and body weight: Streptozotocin ( $45 \mathrm{mg} / \mathrm{kg} \mathrm{bw}$ ) administration resulted in significant elevation of glucose level and reduction in body weight. Administration of two extracts at dose $200 \mathrm{mg} / \mathrm{kg}$ bw administered for 21 days were able to correct this aberration significantly $(p<0.01)$. The results of all the formulations tested are presented in Fig (1).

\section{(a) Body weight}

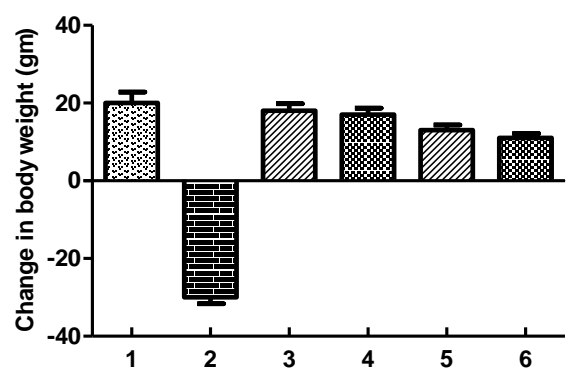

(b) Fasting blood glucose

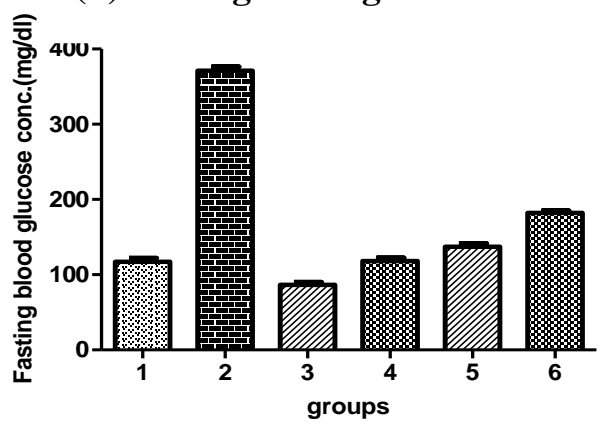

Fig. (1): Effects of rosemary and chamomile leaves extract on (a) body weight and (b) FBG in STZ- induced diabetic rats. Data were given as mean \pm SE for six animals in each group. Statistical analysis was carried out by one way analysis of variance (ANOVA) followed by Students $t$-test. ${ }^{\star} P<0.01$ Results of Gr.1was compared with Gr. 2 and Gr. 2 results with Gr. 3 to 6 .

Effect on protein: There was a marked decrease in the total protein content of untreated diabetic group $(p<0.01)$ when compared with that of the control group. The two herbal formulations were able to correct this metabolic disturbance significantly as given in Fig. (2a).

Effect on $\boldsymbol{\alpha}$-amylase: There was a marked decrease in the serum amylase activity of untreated diabetic group $(p<0.01)$ when compared with that of the control group. The two extracts were able to correct this metabolic disturbance significantly as given in Fig. (2b).

Effect on lipids: Total cholesterol and Triacylglycerol levels were found to be significantly $(p<0.01)$ increased in the vehicle treated diabetic group in comparison with the control group. Treatment with two formulations for a 21 days significantly attenuated $(p<0.01)$ the elevated total cholesterol and Triacylglycerol levels in comparison with the vehicle treated diabetic rats given in Fig. (2c, d).

Effect on TAC: As shown in Fig.(2e), the TAC Level which represents antioxidant enzymes such as superoxide dismutase, Catalase, and glutathione peroxidase; macromolecules such as albumin, ceruloplasmin, and ferritin; and array of small molecules, including ascorbic, $\alpha$-tocopherol, $\beta$ carotene, reduced glutathione, uric acid and Bilirubin, was significantly decreased $(p<0.01)$ in the diabetic group as compared to normal and ongoing treatment with the two herbal extracts the TAC Level was significantly increased compared to normal treated groups.
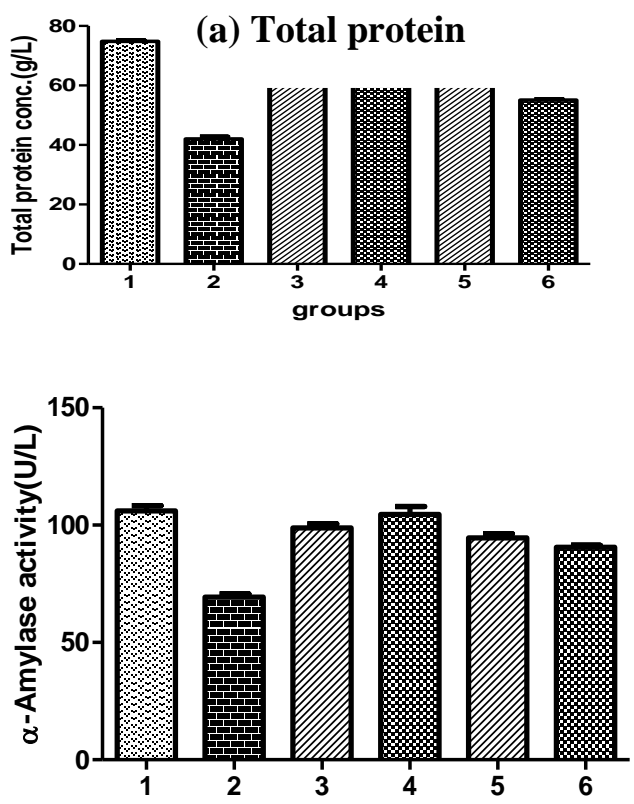

(b) $\alpha$-amylase

(c) Total cholesterol 


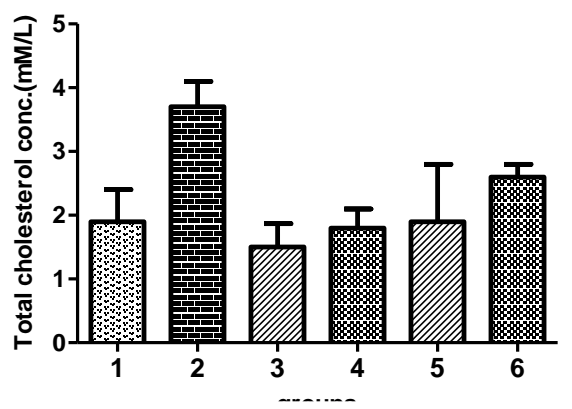

(d) Triglyceride

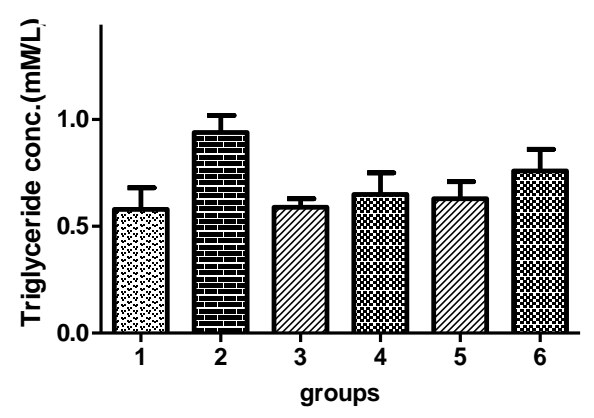

(e) Total antioxidant capacity

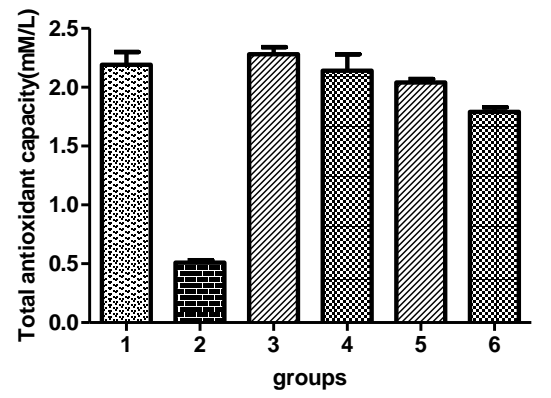

Fig. (2): Effects of rosemary and chamomile leaves extract on some biochemical parameters in STZinduced diabetic rats. Data were given as mean \pm SD for six animals in each group. Statistical analysis was carried out by one way analysis of variance (ANOVA) followed by Students $t$-test. $P<0.01$ Results of Gr. 1 was compared with Gr. 2 and Gr. 2 results with Gr. 3 to 6 .

\section{DISCUSSION AND CONCLUSION}

The world is facing an explosive increase in the incidence of diabetes mellitus and cost effective complementary therapies are needed. Although insulin has become one of the most important therapeutic agents known to medicine, there is a continuing effort to find insulin substitutes, secretagogues, or sensitizers from synthetic or plant sources for the treatment of diabetes (Ali et al., 2009). In the present study, the aqueous extracts of rosemary and chamomile were investigated for its antidiabetic activity in diabetic rats. Animal models of diabetes are increasingly being used for pathophysiology and pharmacological studies of diabetes mellitus. Advantages of animal studies in the examination of alternative medicines and their efficacy include the ability to define experimental conditions more tightly and to undertake more detailed studies of the biologic effects of the agents being used. Streptozotocin-induced hyperglycemia in rodents is considered to be a good experimental model since it is less toxic than other chemical agents inducing diabetes. The mechanisms by which Streptozotocin brings about its diabetic state include selective destruction of pancreatic insulin secreting $\beta$ cells, which make cells less active and lead to poor glucose utilization by tissues (Lenzen, 2008).

In the present study, the intraperitonially administration of STZ to normal rats effectively induced diabetes as reflected by glucoseurea, hyperglycemia and body weight loss. The two plants extracts treatment showed as significant Aantihyperglycemic effects. But none of these extracts could produce any hypoglycemic effect in normal rats. The experimental results indicated that the rosemary and chamomile extracts exhibited a potent blood glucose lowering property in diabetic rats. The capacity of plant extracts to decrease the elevated blood glucose level to normal glycogenic level is an essential trigger for the liver to revert to its normal homeostasis during experimental diabetes. These results suggested that one of the possible mechanisms by which chamomile and rosemary extract (Li et al., 2006) bring about their Aantihyperglycemic action is due to inhibition of hepatic glycogen degradation.

Induction of diabetes with STZ is associated with the characteristic loss of body weight, which is due to increased muscle wasting and due to loss of tissue proteins (Kato et al., 2008). Diabetic rats treated with the plant extracts showed significant gain in body weight as compared to the diabetic control, which may be due to its protective effect in controlling muscle wasting (i.e. reversal of gluconeogenesis and glycogenolysis) and may also be due to the improvement in insulin secretion and glycogenic control. Insulin deficiency leads to various metabolic aberrations, the animals namely increase blood glucose, decreased protein content and increased levels of cholesterol and triglyceride. (Tenpeand Yeole, 2009). 
Increased free radical generation and oxidative stress are hypothesized to play an important role in pathogenesis of diabetes and it's late to play an important role in pathogenesis of diabetes and its late complications. Free radicals are formed disproportionately in diabetes by glucose auto oxidation, thus result in consumption of antioxidant defenses which lead to disruption of cellular function and oxidative damage to membranes and enhance susceptibility to lipid peroxidation. (Baynes, 1991). B $\beta$-cell is particularly sensitive to damage by free radicals because of their low level of free radical scavenging enzymes that leads to hyper glycaemic condition. Reduced oxidative stress due to reduced hyperglycemic status in diabetic condition had been observed in experimental animals following the administration of certain natural compounds (Sanerset al., 2001)

In the present study, results of TAC revealed the efficacy of two plant extracts in the modulation of oxidative stress associated with diabetic rats. The significant antidiabetic activity of formulations may be due to inhibition of free radical generation and subsequent tissue damage induced by STZ or potentiating of plasma insulin effect by increase either pancreatic secretion of insulin from existing beta cells or its release from bound form as indicated by significant improvement in glucose and protein level because insulin inhibit gluconeogenesis from protein.

In accordance to the present findings Tsai et al., (2007) reported that Labiatae family including rosemary, oregano, marjoram and thyme, revealed significant DPPH (2,2-diphenyl-1picrylhydrazyl)inhibitory activity and Phenolic compounds were considered as the main contributors responsible for the free radical scavenging activity) of the total hexane extract of many antidiabetic plants (Tsai et al., 2005. Bakırelet al., 2008 attributed the anti-diabetic, anti-inflammatory anti-tumor and ant proliferative effect of many Labiatae species, to their essential oil which are composed of mono, sesquiterpenes, Phenolic compounds and flavonoides such as Cinnamic acid, caffeic acid and rosmarinic acid.

Several studies have investigated the changes in pancreatic secretion function using STZ-induced diabetes models, (Duanet al., 1989; Aughsteen et al., 1996; Anderson and Bevan, 1992; Yoshihiko et al., 2007) and all found that amylase activity was significantly decreased in STZ-treated rats. Moreover, some studies documented that insulin stimulated the transcription and translation of amylase mRNA and it is therefore likely that insulin stimulates amylase secretion (Patel et al.,2004). In the present study, $40 \mathrm{mg} / \mathrm{kg}$ of STZ markedly decreased serum amylase activity in diabetic groups, thus agreeing with these previous studies, also there was decrease in normal treated group with rosemary compared to normal group. In parallel results (McCue and Shetty 2004) illustrated that the porcine pancreatic amylase was inhibited in vitro in the presence of herbal extracts containing rosmarinic acid (RA-Labiatae) and the extent of amylase inhibition correlated with increased concentration of RA. The anti- amylase inhibitory activity may be due to the ability of $P$ phenolic compounds to interact with and / or inhibit proteins enzymes (Rohn et al., 2002). The same authors added that Phenolic substances that are able to form quinines (such as caffeic acid, chlorogenic acid, Gallic acid, etc) are more reactive than those Pphenolic that cannot form quinines and suggested that semiquinones formed may react with amino acid side chains and free thiol groups on the enzyme.

\section{CONCLUSION:}

The present study was conducted to evaluate the antidiabetic activity of two marketed polyherbal formulations. After completion of the study protocol, it was found that with test and standard control treatment, the serum level of glucose, cholesterol, triglycerides, protein, $\alpha$-amylase and TAC improved significantly $(p<0.01)$ as compared to diabetic control, and in comparative evaluation the two plants found to be safe as they did not shown any sign of acute toxicity. The formulation rosemary was found to be more efficacious as compare to chamomile. Hence, the data of present study provide impetus for further molecular and mechanistic studies on the therapeutic action of rosemary and Chamomile extracts, before they can be considerable as a possible insulin replacement or adjuvant in the management of diabetes mellitus. Further investigations on identification of the active principles and their mode of action are needed to unravel the molecular mechanisms involved in the observed effects.

\section{REFERENCES}

Ali L, Azad K, Hassan Z, Mosiluzzaman M, Nahar N, Nasreen, T, Nur-e-Alam $\mathrm{M}$ and Rokeya B. Characterization of the hypoglycemic effects of Trigonella foenum-graecum seed. Plant med. August 2009; 61: 358-60. 
Aughsteen A, Kataoka $\mathrm{K}$ and Shair SA. Correlative morphometric and biochemical study on pancreatic amylase in normal and Streptozotocin-diabetic rats. Pancreas 1996; 13, 295-303

Anderson LC and Bevan CA. Effects of Streptozotocin diabetes on amylase release and cAMP accumulation in rat parotid acinarcells. Arches. Oral. Biol. 1992; 37: 331-336

Bakirel T, Bakirel U, Keleş OU, Ulgen SG and Yardibi H. In vivo assessment of antidiabetic and antioxidant activities of rosemary (Rosmarinus officinalis) in alloxan-diabetic rabbits. J Ethnopharmacology 2008; 116: 64-73.

Baynes JW. Role of oxidative stress in development of complications in diabetes. Diabetes 1991; 40: 405-412.

Chehade JM and Mooradian AD. A Rational Approach to Drug Therapy of Type 2 Diabetes Mellitus. Disease Management. Drugs 2005; 60 (1): 95-113.

Duan RD, Poensgen J, Wicker C, Westrom B and Erlanson AC. Increase in pancreatic lipase and trypsin activity and their mRNA levels in Streptozotocin-induced diabetic rats. Dig. Dis. Sci.1989; 34: 1243-1248.

Kato A, Minoshima Y, Yamamoto J, Adachi I, Watson A, and Nash R. Protective Effects of Dietary Chamomile Tea on Diabetic Complications. J. Agric. Food Chemistry 2008; 56: 8206-8211.

Yoshihiko H, Takuya I, Kaoru Y and Nobuo K. Effects of Hachimi-jio-gan (Ba-Wei-Di-Huang-Wan) on Hyperglycemia in Streptozotocin-Induced Diabetic Rats. Biol. Pharm. Bull 2007; 30(5): 1015-1020.

Lenzen S. The mechanisms of action of alloxan-and Streptozotocin-induced diabetes. Diabetologia 2008; 51: 216-226.

Li WL, Zeng HC, Bukuru $\mathrm{J}$ and De Kimpe N. Natural medicines used in the traditional Chinese medical system for therapy of diabetes mellitus. J. of Ethnopharmacology 2006; 92: 1-21.

Mallick C, Chatterjee K, GuhaBiswas M, and Ghosh D. Antihyperglycemic effects of separate and composite extract of root of Musa paradisiaca and leaf of Coccinia Indica in Streptozotocin induced Diabetic male albino rat. Afr. J. Trad. CAM 2007; 4: 362-371.

Marina T, Onob T, Liub N, Nakamuraa T, Musoc E and Hondaa G. Suppressive Effects of Rosmarinic Acid on Mesangio proliferative Glomerulonephritis in Rats. Nephron 2002; 92:898-904.
McCue $P$ and Shett. Inhibitory effects of rosmarinic acid extracts on porcine pancreatic amylase in vitro. Asia Pacific Journal of Clinical Nutrition 2004; 13 (1): 101106.

McKay DL and Blumberg JB. A review of the bioactivity and potential health benefits of chamomile tea (Matricaria recutita L.). Phytother. Research 2006; 20: 519-530.

Pari L and Uma MJ. Hypoglycemic effect of Musa sapientum L. in alloxan-induced diabetic rats. Journal of Ethnopharmacology 1999; 68: 321-325.

Patel R, Singh J, Yago MD, Vilchez JR, Martinez-Victoria E and Manas M. Effect of insulin on exocrine pancreatic secretion in healthy and diabetic anaesthetized rats. Mol. Cell. Biochemistry 2004; 261: 105-110.

Piedrola G, Novo E, Escober F and Garcia-Robles R. White blood cell count and insulin resistance in patients with coronary artery disease. Annual Endocrinology (Paris) 2001; 62: 7-10.

Rohn S, Rawel HM and Kroll J. Inhibitory effects of plant phenols on the activity of selected enzymes. J. Agric. Food Chemistry 2002; 50: 3566-3571.

Sanders RA, Rauscher FM, and Watkins JB. Effect of quercetin on Streptozotocin- induced diabetic rats .J Biochem. Mol. Toxicology 2001; 15:143-149.

Srivastava JK and Gupta S. Antiproliferative and apoptotic effects of chamomile extract in various human cancer cells. J. Agric. Food Chemistry 2007; 55: 9470-9478.

Tasi PJ, Tsai TH, Yu, CH and Ho SC. Evaluation of Nosuppressing activity of several Mediterranean culinary spices. Food and chemical Toxicology 2007; 45:440447.

Tasi TH, Tasi PJ and Ho SC. Antioxidant and antiinflammatory activities of several commonly used spices. J. Food Science 2005; 70(1):93-97.

Tenpe CR and Yeole PG. Comparative evaluation of antidiabetic activity of some marketed polyherbal formulations in alloxan induced diabetic rats. International Journal of Pharm Tech Research 2009;1(1):43-49.

Vats RK, Kumar V, Kothari A, Mital A and Uma Ramachandran. Emerging targets for diabetes. Curr Science 2005; 88: 241-247.

Weidmann $\mathrm{P}$, Boehlen LM and DE Courten M. Pathogenesis and treatment of hypertension associated with diabetes mellitus. American Heart Journal 1993; 125: 1498-1513. 